Systematic Review/Meta-Analysis

\title{
Platelet-Rich Plasma versus Corticosteroid Injection for the Treatment of Lateral Epicondylitis: A Systematic Review of Systematic Reviews
}

Jordyn A Kemp, MA, ATC', Megan A Olson, MA, ATC', Matthew A Tao, MD², Christopher J Burcal, PhD, ATC ${ }^{1}$

1 School of Health and Kinesiology, University of Nebraska at Omaha, ${ }^{2}$ Department of Orthopaedic Surgery and Rehabilitation, University of Nebraska Medical Center

Keywords: tennis elbow, platelet rich plasma injection, lateral epicondylitis, elbow epicondylitis, corticosteroid injection

https://doi.org/10.26603/001c.24148

\section{International Journal of Sports Physical Therapy}

Vol. 16, Issue 3, 2021

\section{Background}

Lateral epicondylitis (LE) is one of the most commonly reported musculoskeletal disorders in the upper extremity. The mechanism of LE is repetitive motion that causes a strain of the extensor tendons. This consequently causes pain and tendinosis at the tendinous attachment site on the lateral epicondyle. Most cases of LE are treated nonoperatively with a variety of interventions, such as injections.

\section{Purpose}

The aim of this systematic review (SR) is to synthesize the current evidence on the efficacy of platelet rich plasma (PRP) injections versus corticosteroid (CS) injections as treatment interventions for LE.

\section{Study Design}

Systematic Review

\section{Methods}

Online databases were searched from database inception to February 24th, 2020 for relevant SR's evaluating PRP vs. CS injections as treatment methods for LE. Two independent researchers searched and screened for articles that were systematic reviews that directly compared PRP to CS injections for LE.

\section{Results}

A total of five SR's were included in this review that were published between 2016 and 2020. CS injections were more efficacious for short-term pain relief, and PRP injections were more efficacious for long-term pain relief and improved function.

\section{Conclusion}

PRP injections appear to be a more effective long-term treatment option than CS injections for those with LE who did not respond to conservative management.

\section{Level of evidence}

1

\footnotetext{
a Corresponding Author:

Christopher Burcal 


\section{BACKGROUND}

Lateral epicondylitis (LE) is one of the most commonly reported non-traumatic musculoskeletal disorders in the upper extremity. ${ }^{1-3}$ First defined in 1873 , Runge proclaimed it "a chronic, symptomatic degradation involving the wrist extensor tendons on their attachment at the lateral epicondyle of the humerus". ${ }^{4}$ While debate exists about the exact mechanism for this injury, many researchers believe that it is due to the repetitive motions that cause strain to the origins of the extensor tendons at the lateral epicondyle. ${ }^{3,5,6}$ Most commonly affected is the extensor carpi radialis brevis (ECRB), but other muscles such as the supinator, extensor carpi radialis longus, extensor digitorum, extensor digiti minimi and extensor carpi ulnaris can be involved. ${ }^{4}$ The ECRB is particularly susceptible to the tissue degeneration associated with LE given the relative avascularity of the undersurface. ${ }^{7}$ Lateral epicondylitis was given the nickname "tennis elbow" due to repetitive arm movements of the extensors and supinators that tennis demands. An estimate of $5-10 \%$ of all patients reporting LE actually play tennis, indicating that LE is attributed to additional overuse mechanisms relative to the upper limb, such as playing an instrument, consistent typing or repetitive manual work. . $^{2,6}$

The prevalence rates of LE in the general population range from $1-3 \%$, in patients ranging from $30-55$ years old. ${ }^{1-3,8-10}$ Risk factors for developing LE include increased age, elevated body mass index, oral corticosteroid use, history of smoking, and history of additional tendinopathies such as a rotator cuff disease or De Quairvain's syndrome. ${ }^{9}$ The dominant arm is more frequently affected than the non-dominant arm. ${ }^{9,11}$ Most researchers report no gender based differences among the reported cases of LE, but several studies have indicated a slightly higher rate in female patients. ${ }^{6,8,12}$

Common symptoms of LE are lateral elbow pain, pain with active wrist extension, weakened grip strength, and some report mild disability or disuse of the affected limb. ${ }^{2}$ Mild daily activities such as shaking hands, turning door knobs, or grasping objects for a moderate length of time have been known to aggravate symptoms. ${ }^{9}$ Although the medical suffix '-itis' implies an 'inflammation of', most clinicians agree that LE is a more chronic, degenerative pathology. A failure of normal tendon microtrauma and subsequent repair has been expressed in the histological findings associated with epicondylitis. ${ }^{1,13}$ Histopathologic studies have shown an insufficient amount of inflammatory cells, such as neutrophils or macrophages, in tissue samples, suggesting that the term 'tendinosis' may be more appropriate for this condition. ${ }^{8}$

Current treatments for LE include both nonoperative and operative interventions. Nonoperative methods include rest, bracing, physical therapy, therapeutic ultrasound, extracorporeal shockwave therapy, dry needling, local corticosteroid (CS) injections, injections of biological agents such as platelet-rich plasma (PRP) and autologous blood $(\mathrm{AB})$, botulinum toxin injections $(\mathrm{BT})$, rest, bracing or acupuncture. $2,4,6,8$ Surgical interventions are not commonly utilized, as physicians frequently deem LE a self-limiting injury, and are reserved for patients who have been unresponsive to nonoperative methods for approximately 6-12 months. ${ }^{4,9}$ It has been estimated that approximately $90 \%$ of all reported cases can be treated nonoperatively. ${ }^{4}$ Surgery typically involves debridement of the ECRB tissue through open, arthroscopic, and percutaneous techniques. ${ }^{9}$ Open surgical approaches often use a suture-anchor repair of the ECRB tendon to the lateral epicondyle. ${ }^{14}$ Other surgical techniques include denervation of the lateral epicondyle, a V-Y slide of the common extensor tendon, extensor fasciotomy, intra-articular repairs, epicondylar resection with anconeus transfer, and lengthening of the extensor tendons. 4

Historically, the injection of choice for LE has been CS injection to target pain relief and improve function and strength due to their anti-inflammatory properties. Most agree that LE is not an inflammatory condition, so the question remains on whether this treatment is optimal and ultimately beneficial. In fact, some believe such intra-tendinous CS injections may be detrimental to the long-term healing process and may compromise the tensile strength of the target tissue. ${ }^{1}$ More recently proposed is the use of biologic agents to stimulate the body's natural healing process on the involved tissue. $\mathrm{AB}$, or whole blood, was first understood to trigger an inflammatory reaction and promote tissue healing with cellular and humoral mediators. ${ }^{3}$ Since PRP is derived from the patient's whole blood, it consists of higher concentrations of platelets and potential healing factors released from alpha and delta granules. Among others, PRP contains transforming growth factor beta (TGF$\beta$ ) and vascular endothelial growth factor (VEGF), which promote recruitment, proliferation, and differentiation of cells involved in tissue repair and regeneration. ${ }^{2,3,6}$ Previous systematic reviews (SR's) have assessed the effects of PRP injections compared to CS injections, and the purpose of this review was to synthesize previous SR results into a systematic review of SR's evaluating PRP injections compared to CS injections in the treatment of LE.

\section{METHODS}

\section{SEARCH STRATEGY}

The focus of this review was on previous SR considering the comparison of CS injections to PRP injections as a treatment for LE. The search strategy for this review included articles obtained from the Cochrane Library, ProQuest, and PubMed databases from date of inception to February 24th, 2020. The search key-words include the terms "platelet-rich plasma", "corticosteroid" and "lateral epicondylitis".

\section{INCLUSION AND EXCLUSION CRITERIA}

Studies were included from the search if they matched the following criteria: (1) designed as a SR; (2) written in English, (3) involved a direct comparison of CS to PRP for the intervention. Studies were excluded based on the following criteria: (1) involved interventions other than the direct comparison of CS and PRP; (2) grouped PRP into treatments involving $\mathrm{AB}$ or whole-blood platelets; (3) used the broad term "regenerative therapies" or "regenerative injections" without specification to what the specific intervention was. 
Table 1: Assessment of Multiple Systematic Reviews 2 (AMSTAR 2)

\begin{tabular}{|c|c|c|c|c|c|}
\hline & $\begin{array}{l}\text { Arirachakaran } \\
\text { et al. } 2015^{8}\end{array}$ & $\begin{array}{l}\text { Ben-Nafa } \\
\text { et al. } 2018^{1}\end{array}$ & $\begin{array}{l}\text { Huang } \\
\text { et al. } \\
2019^{6}\end{array}$ & $\begin{array}{l}\text { Li et } \\
\text { al. } \\
2019^{2}\end{array}$ & $\begin{array}{l}\text { Tang } \\
\text { et al. } \\
2019^{3}\end{array}$ \\
\hline $\begin{array}{l}\text { Did the research questions and inclusion criteria for } \\
\text { the review include the components of PICO? }\end{array}$ & Yes & Yes & Yes & Yes & Yes \\
\hline $\begin{array}{l}\text { Did the report of the review contain an explicit } \\
\text { statement that the review methods were established } \\
\text { prior to the conduct of the review and did the report } \\
\text { justify any significant deviations from the protocol? }\end{array}$ & Yes & Yes & Yes & Yes & Yes \\
\hline $\begin{array}{l}\text { Did the review authors explain their selection of the } \\
\text { study designs for inclusion in the review? }\end{array}$ & Yes & Yes & Yes & Yes & Yes \\
\hline $\begin{array}{l}\text { Did the review authors use a comprehensive } \\
\text { literature search strategy? }\end{array}$ & Partial Yes & Partial Yes & $\begin{array}{l}\text { Partial } \\
\text { Yes }\end{array}$ & $\begin{array}{l}\text { Partial } \\
\text { Yes }\end{array}$ & $\begin{array}{l}\text { Partial } \\
\text { Yes }\end{array}$ \\
\hline $\begin{array}{l}\text { Did the review authors perform study selection in } \\
\text { duplicate? }\end{array}$ & Yes & Yes & Yes & Yes & Yes \\
\hline $\begin{array}{l}\text { Did the review authors perform data extraction in } \\
\text { duplicate? }\end{array}$ & Yes & Yes & Yes & Yes & Yes \\
\hline $\begin{array}{l}\text { Did the review authors provide a list of excluded } \\
\text { studies and justify the exclusions? }\end{array}$ & Yes & Yes & Yes & Yes & Yes \\
\hline $\begin{array}{l}\text { Did the review authors describe the included studies } \\
\text { in adequate detail? }\end{array}$ & Yes & Yes & Yes & Yes & Yes \\
\hline $\begin{array}{l}\text { Did the review authors use a satisfactory technique } \\
\text { for assessing the risk of bias (RoB) in individual } \\
\text { studies that were included in the review? }\end{array}$ & Partial Yes & Partial Yes & $\begin{array}{l}\text { Partial } \\
\text { Yes }\end{array}$ & $\begin{array}{l}\text { Partial } \\
\text { Yes }\end{array}$ & $\begin{array}{c}\text { Partial } \\
\text { Yes }\end{array}$ \\
\hline $\begin{array}{l}\text { Did the review authors report on the sources of } \\
\text { funding for the studies included in the review? }\end{array}$ & Yes & Yes & Yes & Yes & No \\
\hline $\begin{array}{l}\text { If meta-analysis was performed did the review } \\
\text { authors use appropriate methods for statistical } \\
\text { combination of results? }\end{array}$ & Yes & No M-A & Yes & Yes & Yes \\
\hline $\begin{array}{l}\text { If meta-analysis was performed, did the review } \\
\text { authors assess the potential impact of RoB in } \\
\text { individual studies on the results of the meta-analysis } \\
\text { or other evidence synthesis? }\end{array}$ & Yes & No M-A & No & Yes & Yes \\
\hline $\begin{array}{l}\text { Did the review authors account for RoB in individual } \\
\text { studies when interpreting/ discussing the results of } \\
\text { the review? }\end{array}$ & Yes & Yes & No & Yes & Yes \\
\hline $\begin{array}{l}\text { Did the review authors provide a satisfactory } \\
\text { explanation for, and discussion of, any heterogeneity } \\
\text { observed in the results of the review? }\end{array}$ & Yes & Yes & Yes & Yes & Yes \\
\hline $\begin{array}{l}\text { If they performed quantitative synthesis did the } \\
\text { review authors carry out an adequate investigation } \\
\text { of publication bias (small study bias) and discuss its } \\
\text { likely impact on the results of the review? }\end{array}$ & Yes & No M-A & Yes & Yes & Yes \\
\hline $\begin{array}{l}\text { Did the review authors report any potential sources } \\
\text { of conflict of interest, including any funding they } \\
\text { received for conducting the review? }\end{array}$ & Yes & Yes & Yes & Yes & No \\
\hline $\begin{array}{l}\text { Overall Methodological Quality } \\
(\mathrm{CL}=\text { critically low, } \mathrm{L}=\text { low, } \mathrm{M}=\text { =moderate, } \mathrm{H}=\text { high) }\end{array}$ & $\mathrm{H}$ & $M$ & L & $\mathrm{H}$ & $M$ \\
\hline
\end{tabular}

\section{RISK OF BIAS ASSESSMENT}

Two authors (JK, MO) independently assessed the risk of bias for each SR. There was no involvement of third-party analysis for the review of sources of bias. Any disagreement was discussed between the two authors until they came to a consensus.

\section{QUALITY ASSESSMENT}

Table 1 summarizes A MeaSurement Tool to Assess systematic Reviews 2 (AMSTAR 2) results for each SR article included in this review. The AMSTAR 2 tool is a critical appraisal tool for health professionals to assess the quality of conduct of SR. ${ }^{15}$ Rather than focusing on an overall score, the AMSTAR 2 emphasizes the presence of critical flaws 


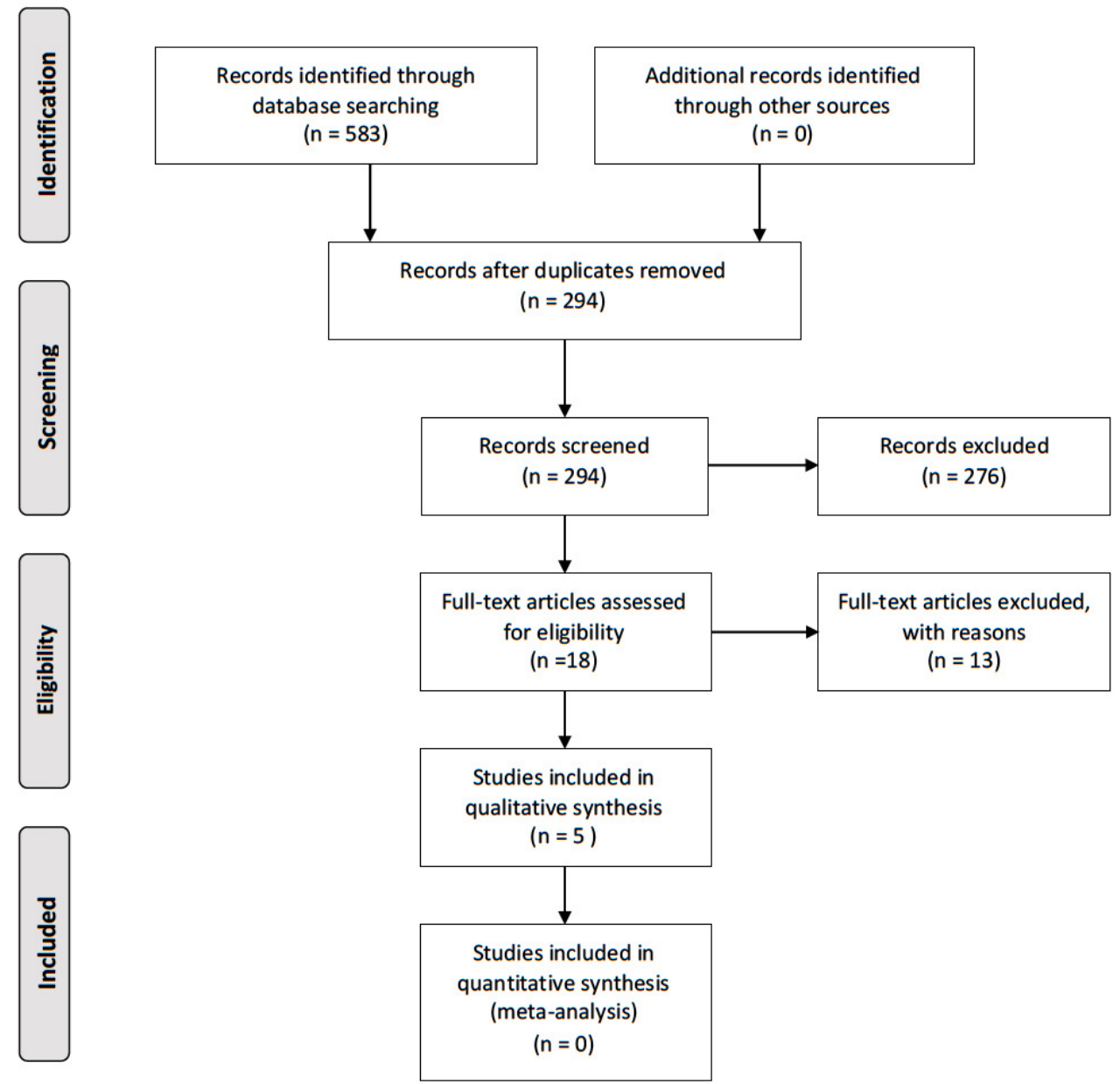

Figure 1: PRISMA Diagram

or weaknesses within the critical domains, which may influence the risk of bias. ${ }^{15}$ We also calculated the corrected covered area (CCA) index in order to account for overlap in the included original studies in each individual SR. We calculated and interpreted the CCA using the methods and guidelines suggested by Pieper et al. ${ }^{16}$ In brief, the overlap of original studies identified and included in the individual SRs can be seen in Supplementary Table 1. The CCA represents the \% overlap of included original studies compared to a corrected total amount of possible original studies; higher values $(\geqslant 15 \%)$ indicate more significant overlap. ${ }^{16,17}$

\section{RESULTS}

Figure 1 shows the PRISMA flow diagram for the search of the SR. The primary search resulted in 583 articles. After all duplicates were removed, a total of 294 articles were screened. Based on abstract searches, title searches, and filtering for SR, the number of full text articles that qualified were 18. Out of those 18 articles, there were five SR that met the inclusion and exclusion criteria for this review and can be seen in Appendix A.

\section{STUDY DESIGN CHARACTERISTICS OF INCLUDED SYSTEMATIC REVIEWS}

All five of the included SR used Medline to search for their included literature. Other common databases that were used among the included SR were: Embase $(n=4)$, Cochrane Library $(n=4)$, Scopus $(n=2)$, and Web of Science $(n=2)$. Several additional databases were searched in the individual studies. The number of databases used ranged from two to 11. These five SR's included a range of five to 20 randomized control trials. Additionally, all of these SR's evaluated multiple patient reported outcome measures (PRO's) for the treatment of LE. These PRO's included the Visual Analogue Scale for pain (VAS) $(n=5)$, Disabilities of Arm, Shoulder and Hand (DASH) $(n=5)$, Pain Pressure Threshold (PPT) $(n=2)$, Patient-Related Tennis Elbow Evaluation (PRTEE) $(n=3)$, modified Nirschl score for pain (MNS) $(n=1)$, and modified MAYO score (MMS) $(\mathrm{n}=1)$.

\section{AMSTAR 2 AND CCA RESULTS}

The AMSTAR 2 methodological assessment showed varying qualities amongst the five included SR's. The results indicated two high quality SR, two moderate quality SR, and one low quality SR. All five reviews included a statement of publication bias, a comprehensive literature search, a 
list of included and excluded studies, scientific evaluation of the quality of the included studies, documentation and evaluation of the specific quality of the included studies, and appropriate methods to assess the data and formulate conclusions. The one study that was low quality lacked an appropriate acknowledgement or assessment of risk of bias during synthesis and discussion. The CCA for all five included SRs was $28.3 \%$, indicating a high amount of overlap among the original included studies.

\section{LATERAL EPICONDYLITIS TREATMENT RESULTS}

The main treatment interventions used in all of these SR's were CS injections in comparison to PRP injections. Two of the studies included a third treatment intervention, separately comparing $\mathrm{AB}$ to $\mathrm{CS}$ and $\mathrm{PRP}$. Four out five studies revealed that $C S$ injections proved beneficial for short-term (2-8 weeks) pain relief and improved function, while PRP injections proved more beneficial for long-term ( $>8$ weeks) pain relief and function. ${ }^{1-3,6}$ One SR involving $A B$ concluded it was superior to CS in all categories; however, PRP was significantly better than both $\mathrm{AB}$ and $\mathrm{CS}$ in all categories. ${ }^{3}$ Another study involving $\mathrm{AB}$ revealed that $\mathrm{PRP}$ was the best treatment for reducing pain, whereas $A B$ injection was the best treatment for improving disabilities scores and increasing PPT. ${ }^{8}$

\section{DISCUSSION}

The purpose of this study was to consolidate current evidence on the effectiveness of PRP injections versus CS injections for the treatment of LE. This study assessed SR to determine the best comparative intervention for treating epicondylitis. This systematic review of SRs conveyed evidence to support the use of PRP injections as an effective, long-term treatment option over CS injections for those with LE. Collectively, all five SR's assessed pain through VAS and function through DASH scores as primary PRO. ${ }^{1-3,6,8}$ Three out of five SR's evaluated Patient-Related PRTEE scores, providing condition-specific PRO results. ${ }^{1,3,8}$ The evidence suggests that PRP is more effective than CS longterm in all of the investigated PRO including pain, function, disability, and PPT. ${ }^{1-3,6,8}$ In contrast, one study reported that a third intervention, autologous blood $(\mathrm{AB})$, was more effective for improving disabilities scores and increasing pressure threshold within and after two months. ${ }^{8}$ Congruence and consistency of results existed among all 5 SR in favor of using PRP injections for optimal long-term outcomes.

This SR aimed to observe the short- and long-term effects of CS injections to PRP injections in the treatment of LE, without specification to the observable outcomes. Many of the studies in this review observed pain (VAS) and function (DASH) as their main outcomes, with few studies focusing on additional outcomes such as strength. One SR yielded results that showed there was no difference in strength between the PRP and CS injection groups before or after the treatment intervention. ${ }^{3}$ The reviews included in this SR had minor inconsistencies regarding measurable outcomes, duration of symptoms, and the timing of followups amongst participants. The variability of reported PROs is notable but ultimately does not detract from the overall conclusion of this SR. Evidence regarding the comparison of CS injections to PRP injections can be applied to other overuse injuries that present with impaired function and significant pain.

A major inconsistency in the literature regarding PRP injections is the controversy between factors of the injection, including optimal volume, timing, injection technique, and the quality of the PRP preparations. Amongst the studies in this review, the volume of PRP concentrations range from 1.0-5.0 mL, while some studies stated the amount of autologous blood taken and the time it was centrifuged. Many trials in these studies did not specify the amount of PRP or the concentration relative to resting levels of platelets in the patient's blood. Previous studies also fail to report whether the plasma sample contains leukocyte-rich or leukocyte-poor PRP formulations. Castillo et al. ${ }^{18}$ compared three centrifuge systems and reported significant differences in white blood cell (WBC) and growth factor concentrations in all three samples. These factors could indicate varying amounts of healing factors within different plasma concentrations obtained from different separation systems. Multiple studies reported using centrifuge systems but gives no indication to the concentration of platelets derived from the blood or utilized in the injection. Castillo et al. ${ }^{18}$ also reported obtaining similar volumes of PRP from all three of the centrifuge systems studied, despite each system utilizing different volumes of whole blood.

Three techniques seem to be most commonly reported in the reviewed literature for delivering the PRP concentration to the tissue: (1) ultrasound guided injection, in which a clinician injects the most vulnerable or degraded tissue with the injection; (2) peppering, in which the clinician injects small amounts of the PRP concentration in multiple sites around the target tissue to enhance the effects of the PRP; (3) blind injection at the point of maximal tenderness. Future research should aim to resolve such inconsistencies in PRP injections, including the outcomes from different PRP concentrations to observe the minimum amount of platelet concentration required for the desired effects. Creating optimal guidelines for PRP injections would help both clinicians and researchers investigating the effects of PRP injections. Despite the inconsistencies in reporting preparation techniques, injection techniques, and concentrations of PRP, the results remain in favor of PRP as a long-term treatment for LE. Further studies should aim to identify the role that each of these factors play into the efficacy of PRP in the treatment of LE.

Inconsistencies involving CS injections were similar to the previously mentioned inconsistencies of PRP injections. Many of the included SRs contained studies with several different reported CS injections, if not a combination of two CS drugs. Whether there are benefits to an individual CS drug versus a combination of CS drugs is unknown. ${ }^{19} \mathrm{Ad}-$ ditionally, there are several reported injections involving anesthetics as well, aiming to alleviate both the symptoms of LE and the potential pain caused by the injection. The three previously mentioned injection delivery techniques were utilized amongst all the included reviews. Incongruity, or lack of reporting entirely, of the delivery technique was observed in all the included SR. Although the evidence still 
supports the use of CS for short-term improvements, respectively, a lack of consensus remains on the optimal technique.

Injection therapies such as CS and PRP have several useful properties that are applicable to clinical practice. However, their proposed mechanisms and effects on the tissue differ significantly. Injections containing autologous material such as PRP have been proven to facilitate tissue healing through higher concentrations of growth factors, which promote tissue repair and have a direct influence on angiogenesis and inflammation. ${ }^{2}$ The effects of PRP treatments are not observed as acutely when compared to the effects of CS injections, yet, they have a longer duration of pain relief and improved function. ${ }^{1-3,6,8}$ The use of CS injections have been identified in literature to provide short-term relief for a multitude of inflammatory pathologies such as bursitis, tendinitis, and osteoarthritis (OA). ${ }^{20}$ Some studies have concluded CS injections provide short-term pain relief and slightly improved function, though these effects decrease over time. Furthermore, receiving multiple CS injections may be a risk factor for surgical intervention and possible future surgical revision. ${ }^{21}$ Multiple sources discuss the possible deleterious effects that can occur with chronic CS use, ${ }^{21}$ which has yet to be observed with PRP injections. ${ }^{1,22,23}$ Because the underlying pathology of LE is degenerative in nature, PRP injections may provide more benefit due to their potential regenerative properties.

The overall quality of the evidence presented in this SR is moderate-to-high quality based on our assessment of bias using the AMSTAR 2 tool (Table 1). The included reviews all investigated the same interventions of PRP and CS injections. Two out of the five reviews included a third comparative treatment $(\mathrm{AB})$. However, the specific PRP preparation methods and specific CS injection drug type varied across studies. In the reporting process, all of the reviews included in this SR completed a comprehensive literature search, had duplicate selection and data extraction, provided a list of included and excluded studies, used scientific evaluation methods to assess article quality and combine their findings to form conclusions, and evaluated publication bias. Additionally, $80 \%$ of the included studies stated the potential conflicts of interest in their reviews. One potential explanation for the consistency in the cumulative findings of all five SR's comes from the high CCA score, which indicates a high degree of overlap of original studies that were included in the individual SR (Supplementary Table 1). Based on these findings, it appears that the most recent SR on this topic by Tang et al. ${ }^{3}$ builds on the findings of Arirachakaran et al. ${ }^{8}$ There is also significant overlap between the SR conducted by Li et al. ${ }^{2}$ and Huang et al., ${ }^{6}$ which is not surprising considering how close together the two are in their search dates.

The evidence evaluated in this SR shows a strong consensus that PRP injections are superior to CS injections for long-term treatment of LE. However, these results have certain limitations. One limitation is the variability of contents and preparation for both PRP and CS injections in all studies. It is important to note that even though certain methodological details were inconsistent across the studies, all 5 reviews yielded similar results. Furthermore, these limitations we have identified are primarily due to reporting practices. Therefore, the authors encourage authors of future clinical trials utilizing injection therapies to include information regarding their injection parameters (e.g. dose, centrifuge time, drug concentrations, injection technique).

\section{CONCLUSION}

In conclusion, the results of this systematic review of SR demonstrates that PRP is a superior long-term treatment option for LE when compared to CS, while CS has been proven to create a short-term decrease in pain. Further research should be conducted on specific PRP procedures such as preparation and injection methods, platelet concentrations, and centrifugation time to determine whether certain methodological protocols are more effective than others at decreasing pain and improving function in LE. Furthermore, longer follow-up periods should be used in future research to understand the full extent of the long-term effects of PRP injections, specifically the duration of the effects and if patients require a secondary injection. Based on the evidence presented in these SR's, PRP is an efficacious long-term treatment option for reducing pain and improving function for patients with LE who have otherwise failed conservative management.

\section{CONFLICT OF INTEREST DISCLOSURE}

None of the authors have any conflicts of interest pertinent to this manuscript to disclose.

Submitted: July 09, 2020 CDT, Accepted: February 23, 2021 CDT 


\section{REFERENCES}

1. Ben-Nafa W, Munro W. The effect of corticosteroid versus platelet-rich plasma injection therapies for the management of lateral epicondylitis: A systematic review. SICOT-J. 2018;4:11. doi:10.1051/sicotj/201706 $\underline{2}$

2. Li A, Wang H, Yu Z, et al. Platelet-rich plasma vs corticosteroids for elbow epicondylitis: A systematic review and meta-analysis. Medicine. 2019;98(51):e18358. doi:10.1097/md.0000000000018 $\underline{358}$

3. Tang S, Wang X, Wu P, et al. Platelet - rich plasma vs autologous blood vs corticosteroid injections in the treatment of lateral epicondylitis: A systematic review, pairwise and network meta - analysis of randomized controlled trials. $P M R$.

2020;12(4):397-409. doi:10.1002/pmrj.12287

4. Vaquero-Picado A, Barco R, Antuña SA. Lateral epicondylitis of the elbow. EFORT Open Rev. 2016;1(11):391-397. doi:10.1302/2058-5241.1.000049

5. Bisset L, Paungmali A, Vicenzino B, et al. A systematic review and meta-analysis of clinical trials on physical interventions for lateral epicondylalgia. Br J Sports Med. 2005;39(7):411-422. doi:10.1136/bjs m.2004.016170

6. Huang K, Giddins G, Wu L. Platelet-rich plasma versus corticosteroid injections in the management of elbow epicondylitis and plantar fasciitis: An updated systematic review and meta-analysis. Am J Sports Med. 2019;48(10):2572-2585. doi:10.1177/036354651 $\underline{9888450}$

7. Schneeberger AG, Masquelet AC. Arterial vascularization of the proximal extensor carpi radialis brevis tendon. Clin Orthop Rel Res. 2002;398:239-244. doi:10.1097/00003086-200205000-00033

8. Arirachakaran A, Sukthuayat A, Sisayanarane T, Laoratanavoraphong S, Kanchanatawan W, Kongtharvonskul J. Platelet-rich plasma versus autologous blood versus steroid injection in lateral epicondylitis: Systematic review and network metaanalysis. J Orthop Traumatol. 2016;17(2):101-112. do $\mathrm{i}: 10.1007 / \mathrm{s} 10195-015-0376-5$

9. Lai WC, Erickson BJ, Mlynarek RA, Wang D. Chronic lateral epicondylitis: Challenges and solutions. Open Access J Sports Med. 2018;9(243):243-251. doi:10.2147/oajsm.s160974
10. Shiri R, Viikari-Juntura E. Lateral and medial epicondylitis: Role of occupational factors. Best Pract Res Clin Rheumatol. 2011;25(1):43-57. doi:10.1016/i.b erh.2011.01.013

11. Calfee RP, Patel A, DaSilva MF, Akelman E. Management of lateral epicondylitis: Current concepts. J Am Acad Orthop Surg. 2008;16(1):19-29. d oi:10.5435/00124635-200801000-00004

12. Shiri R, Viikari-Juntura E, Varonen H, Heliovaara $\mathrm{M}$. Prevalence and determinants of lateral and medial epicondylitis: A population study. Am J Epidemiol. 2006;164(11):1065-1074. doi:10.1093/aje/kwi325

13. Ciccotti MC, Schwartz MA, Ciccotti MG. Diagnosis and treatment of medial epicondylitis of the elbow. Clin Sports Med. 2004;23(4):693-705. doi:10.1016/i.cs $\underline{\text { m.2004.04.011 }}$

14. Wang D, Degen RM, Camp CL, McGraw MH, Altchek DW, Dines JS. Trends in surgical practices for lateral epicondylitis among newly trained orthopaedic surgeons. Orthop J Sports Med. 2017;5(10):232596711773057. doi:10.1177/232596711 $\underline{7730570}$

15. Shea BJ, Reeves BC, Wells G, et al. AMSTAR 2: A critical appraisal tool for systematic reviews that include randomised or non-randomised studies of healthcare interventions, or both. BMJ. 2017;358:j4008. doi:10.1136/bmj.j4008

16. Pieper D, Antoine S-L, Mathes T, Neugebauer EAM, Eikermann M. Systematic review finds overlapping reviews were not mentioned in every other overview. J Clin Epidemiol. 2014;67(4):368-375. doi:10.1016/j.jclinepi.2013.11.007

17. Hennessy EA, Johnson BT. Examining overlap of included studies in meta-reviews: Guidance for using the corrected covered area index. Res Synth Methods. 2020;11(1):134-145. doi:10.1002/jrsm.1390

18. Castillo TN, Pouliot MA, Kim HJ, Dragoo JL. Comparison of growth factor and platelet concentration from commercial platelet-rich plasma separation systems. Am J Sports Med. 2011;39(2):266-271. doi:10.1177/0363546510387517

19. Bisset L, Coombes B. Tennis elbow. BMJ Clin Evid. $2011 ; 2011$.

20. Foster ZI, Voss TT, Hatch J, et al. Corticosteroid injections for common musculoskeletal conditions. Am Fam Physician. 2015;92(8):694-699. 
21. Degen RM, Cancienne JM, Camp CL, Altchek DW, Dines JS, Werner BC. Three or more preoperative injections is the most significant risk factor for revision surgery after operative treatment of lateral epicondylitis: An analysis of 3863 patients. J Shoulder Elbow Surg. 2017;26(4):704-709. doi:10.1016/j.jse.201 6.10.022

22. Mohamadi A, Chan JJ, Claessen FMAP, Ring D, Chen NC. Corticosteroid injections give small and transient pain relief in rotator cuff tendinosis: A meta-analysis. Clin Orthop Relat Res.

2017;475(1):232-243. doi:10.1007/s11999-016-5002-1
23. Smidt N, Van Der Windt DA, Assendelft WJ, Devillé WL, Korthals-de Bos IB, Bouter LM. Corticosteroid injections, physiotherapy, or a waitand-see policy for lateral epicondylitis: A randomised controlled trial. Lancet. 2002;359(9307):657-662. do $\mathrm{i}: 10.1016 / \mathrm{s} 0140-6736(02) 07811-\mathrm{X}$ 


\section{SUPPLEMENTARY MATERIALS}

\section{Appendix A}

Download: https://ijspt.scholasticahq.com/article/24148-platelet-rich-plasma-versus-corticosteroid-injection-for-thetreatment-of-lateral-epicondylitis-a-systematic-review-of-systematic-reviews/attachment/61103.docx 